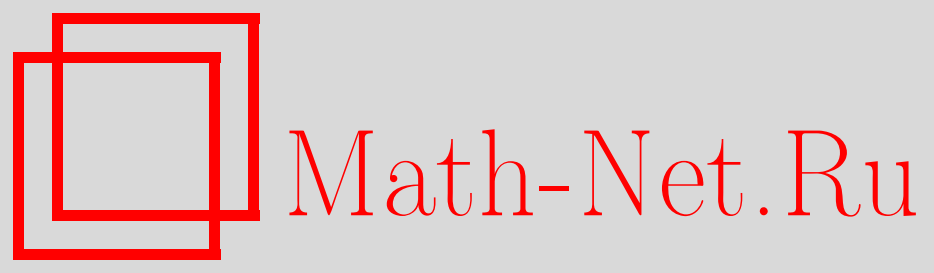

Д. К. Демской, Интегралы открытых двумерных цепочек, ТMФ, 2010, том 163, номер 1, 79-85

DOI: https://doi.org/10.4213/tmf6487

Использование Общероссийского математического портала Math-Net.Ru подразумевает, что вы прочитали и согласны с пользовательским соглашением http://www . mathnet.ru/rus/agreement

Параметры загрузки:

IP: 54.174 .149 .18

26 апреля 2023 г., 16:36:02

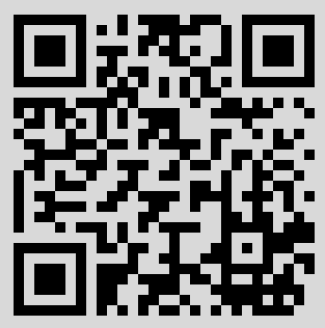




\section{ИНТЕГРАЛЫ ОТКРЫТЫХ ДВУМЕРНЫХ ЦЕПОЧЕК}

Предложена явная формула для интегралов открытой двумерной цепочки Тоды типа $A_{n}$, применимая к различным редукциям этой цепочки. В качестве иллюстрации найдены интегралы цепочки Тоды $G_{2}$. Выявлена также связь между открытой цепочкой Тоды $A_{n}$ и цепочкой Шабата-Ямилова.

Ключевые слова: интегралы, открытая двумерная цепочка Тоды.

\section{1. ВВЕДЕНИЕ}

Наиболее известные представители класса точно решаемых гиперболических систем - двумерные цепочки Тоды

$$
u_{i, t x}=\exp \left\{\sum_{j=1}^{n} A_{j}^{i} u^{j}\right\}, \quad i=1, \ldots, n,
$$

где $\left(A_{j}^{i}\right)$ - матрица Картана простой алгебры Ли. Общий метод интегрирования таких систем был предложен Лезновым и Савельевым [1]. Вариант цепочки (1), соответствующий классической серии $A_{n}$, был известен Дарбу, нашедшему также ее общее решение. Точно решаемые системы обладают несколькими характеристическими свойствами, отличающими их от множества других систем. К ним, в частности, относятся конечность цепочек обобщенных инвариантов Лапласа [2], [3], а также наличие нетривиальных интегралов и обобщенных симметрий. Известно также, что последние две структуры связаны друг с другом посредством дифференциального оператора, отображающего интегралы в симметрии [4]. Давно известно, что системы (1) имеют полные наборы интегралов [5], однако явные формулы для них не были построены, за исключением нескольких особых случаев. В настоящей статье предлагается решение этой задачи для цепочки Тоды $A_{n}$ и ее редукций.

Простейшее гиперболическое интегрируемое уравнение - уравнение Даламбера

$$
w_{t x}=0 .
$$

Однако возможность обобщения записанного в такой форме уравнения на случай уравнений высшего порядка или систем уравнений не очевидна.

*Academia Sinica, Taipei, Taiwan. E-mail: demskoi@math.sinica.edu.tw 
Если ввести новую зависимую переменную $w=\ln u$, то уравнение (2) превращается в уравнение

$$
\operatorname{det}\left(\begin{array}{cc}
u & u_{t} \\
u_{x} & u_{t x}
\end{array}\right)=0,
$$

очевидным обобщением которого будет уравнение $2 n$-го порядка

$$
\operatorname{det}\left(\begin{array}{cccc}
u & u_{t} & \ldots & u_{t \ldots t} \\
u_{x} & u_{t x} & \ldots & u_{t \ldots t x} \\
\vdots & \vdots & \ddots & \vdots \\
u_{x \ldots x} & u_{t x \ldots x} & \ldots & u_{t \ldots t x \ldots x}
\end{array}\right)=0
$$

где $u_{t \ldots t x \ldots x}=\partial_{x}^{n} \partial_{t}^{n} u$. Для краткости запишем его в виде

$$
W_{n+1}(u)=0 .
$$

Это уравнение будет основным для последующих рассуждений. Мы будем далее ссылаться на него как на высшее уравнение Даламбера.

Уравнение (4) можно рассматривать как условие равенства нулю вронскианов:

$$
W\left(u, u_{x}, \ldots, u_{x \ldots x}\right)(t)=0, \quad W\left(u, u_{t}, \ldots, u_{t \ldots t}\right)(x)=0 .
$$

Отсюда можно вывести общее решение

$$
u=X_{1}(x) T_{1}(t)+X_{2}(x) T_{2}(t)+\cdots+X_{n}(x) T_{n}(t),
$$

где $X_{i}, T_{i}$ - произвольные функции.

Очевидно, существуют разные способы записи уравнения (4) как системы уравнений. Дарбу показал, что величины $W_{j}(u)$ удовлетворяют рекуррентному соотношению

$$
\left(\ln W_{j}\right)_{x t}=W_{j-1} W_{j+1} W_{j}^{-2}, \quad W_{0}=1, \quad W_{1}=u .
$$

Изначально это соотношение возникло в связи с изучением инвариантов Лапласа гиперболических уравнений. После введения новых величин

$$
W_{j}(u)=e^{w_{j}}
$$

уравнение (4) преобразуется в систему

$$
w_{j, x t}=e^{w_{j-1}-2 w_{j}+w_{j+1}}, \quad j=1, \ldots, n-1, \quad w_{n, x t}=0,
$$

с граничным условием $w_{0}=0$. Заметим, что можно исключить $w_{n}$ из этой системы с помощью преобразования

$$
w_{n-1} \rightarrow w_{n-1}+\frac{2}{3} w_{n}, \quad w_{n-2} \rightarrow w_{n-2}+\frac{1}{3} w_{n} .
$$

В результате получается система

$$
w_{j, x t}=e^{w_{j-1}-2 w_{j}+w_{j+1}}, \quad j=1, \ldots, n-1, \quad w_{0}=w_{n}=0 .
$$


Систему (9) часто называют открытой (конечной, непериодической) $A_{n-1}$-цепочкой Тоды. Поэтому уравнение $(4)$ и открытая $A_{n}$-цепочка Тоды, возможно, суть простейшие обобщения уравнения Даламбера для уравнений высшего порядка или систем уравнений. Можно спросить, существуют ли другие системы достаточно компактного вида, имеющие отношение к уравнению (4). Очевидно, любая другая система, сводящаяся к уравнению (4), должна также иметь отношение к системе (8). Другой вопрос, представляющий для нас интерес: как структуры, относящиеся к разрешимости высшего уравнения Даламбера, связаны со структурами соответствующих систем уравнений? Очевидно, что интегралы уравнения (4) являются также интегралами системы (8). Удивительно, что формула для скалярного уравнения оказывается много проще, чем формула для соответствующей системы уравнений.

\section{2. ИНТЕГРАЛЫ ОТКРЫТЫХ ЦЕПОЧЕК ТОДЫ}

Рассмотрим некоторые свойства высшего уравнения Даламбера. Мы уже указывали его общее решение, принадлежащее Дарбу, теперь мы хотим показать, что у него также есть $n+n$ независимых интегралов. Заметим, что благодаря симметрии $x \leftrightarrow t$ достаточно представить только $t$-интегралы. Напомним, что функция неизвестных и их производных называется $t$-интегралом, если она удовлетворяет характеристическому уравнению $D_{t} \omega=0$ (детальное изложение см. в работе [2]).

ПреДЛОЖЕНИЕ. Уравнение (4) допускает $n$ независимъх $t$-интегралов вида

$$
\omega_{i}=\frac{W_{n, i}}{W_{n}},
$$

где $W_{n, i}$ - детерминант, полученный из $W_{n}$ заменой $i$-й строки строкой

$$
\left(\partial_{x}^{n} u, \partial_{x}^{n} \partial_{t} u, \partial_{x}^{n} \partial_{t}^{2} u, \ldots, \partial_{x}^{n} \partial_{t}^{n-1} u\right)
$$

Возможно, простейший способ доказать это утверждение - показать, что интегралы (10) становятся функциями одной переменной после подстановки выражения для общего решения (5) в (10). Действительно, после подстановки получаем

$$
\begin{aligned}
W_{n, i} & =W\left(T_{1}, \ldots, T_{n}\right)(t) \sum_{j} X_{j}^{(n)} C_{i j}(x), \\
W_{n} & =W\left(X_{1}, \ldots, X_{n}\right)(x) W\left(T_{1}, \ldots, T_{n}\right)(t),
\end{aligned}
$$

где $C_{i j}(x)$ - алгебраическое дополнение элемента $\left(W\left(X_{1}, \ldots, X_{n}\right)(x)\right)_{i j}$. Поэтому интегралы (4) параметризуются функциями $X_{i}(x)$ следующим образом:

$$
\omega_{i}=\frac{\sum_{j} X_{j}^{(n)} C_{i j}(x)}{W\left(X_{1}, \ldots, X_{n}\right)(x)} .
$$

Независимость этих интегралов следует из самой формулы (10). 
ЗАмЕчАниЕ. Формула (10) дает явное выражение для интегралов не только уравнения (4), но и системы (8). Заметим, что $u=W_{1}=e^{w_{1}}$, а значит, $\omega_{i}$ выражается в терминах одной лишь величины $w_{1}=\ln u$.

Вместо уравнения (4) и системы (8) можно было начать с системы (9). Можно показать, что последняя эквивалентна скалярному уравнению [1]

$$
W_{n}(u)=(-1)^{n(n-1) / 2} .
$$

В этом случае формулы для интегралов должны быть модифицированы:

$$
\omega_{i}=\frac{W_{n, i}^{*}}{W_{n}},
$$

где $W_{n, i}^{*}-$ детерминант, полученный из $W_{n}$ заменой $i$-й строки строкой

$$
\left(\partial_{x}^{n+1} u, \partial_{x}^{n+1} \partial_{t} u, \partial_{x}^{n+1} \partial_{t}^{2} u, \ldots, \partial_{x}^{n+1} \partial_{t}^{n-1} u\right)
$$

Формулы (10) и (11) можно использовать для нахождения интегралов различных цепочек, полученных из уравнения (4). К ним относятся двумерная цепочка Тоды, соответствующая матрице Картана алгебры Ли $A_{n}$, и ее редукции, а также иной вариант двумерной цепочки Тоды, данный формулой (18), и цепочка Шабата-Ямилова (см. ниже). Формулы (10) и (11) выражают интегралы в терминах одной неизвестной переменной и ее производных. Поэтому они верны до тех пор, пока эта переменная не затрагивается редукцией или заменой переменных. В противном случае формулы необходимо соответствующим образом исправить.

Продемонстрируем это на примере цепочки Тоды $G_{2}$ :

$$
p_{t x}=e^{-2 p+q}, \quad q_{t x}=e^{3 p-2 q},
$$

где $w_{1}=p, w_{2}=q$. Известно, что цепочка (12) является редукцией цепочки Тоды $A_{6}$, поэтому ее интегралы даются формулой (11), в которой все смешанные производные следует заменить согласно системе (12). Из формул (7) и (11) имеем

$$
\omega_{i}=\frac{W_{6, i}^{*}\left(e^{p}\right)}{W_{6}\left(e^{p}\right)},
$$

таким образом,

$$
\begin{gathered}
\omega_{6}=q_{x}^{2}+3 p_{x x}-3 q_{x} p_{x}+q_{x x}+3 p_{x}^{2}, \\
\omega_{5}=5 \omega_{6, x}, \quad \omega_{4}=6 \omega_{6, x x}-\omega_{6}^{2}, \quad \omega_{3}=4 \omega_{6, x x x}-3 \omega_{6} \omega_{6, x}, \\
\omega_{2}=\omega_{2}^{*}+\omega_{6, x x x x}-\frac{\left(\omega_{6}^{2}\right)_{x x}}{2}, \quad \omega_{1}=\frac{\omega_{2, x}^{*}}{2},
\end{gathered}
$$


где

$$
\begin{aligned}
\omega_{2}^{*}=2 p_{6} & +2 p_{2} p_{4}-60 p_{1}^{2} p_{2}^{2}+12 p_{1}^{4} p_{2}-28 p_{1}^{3} p_{3}-6 q_{4} p_{2}-6 p_{3} q_{3}-13 q_{2}^{2} p_{1}^{2}+ \\
& +2 p_{2} q_{1}^{4}-2 p_{1}\left(q_{5}-2 p_{5}\right)+14 q_{3} p_{1}^{3}-10 p_{2} q_{2}^{2}+4 q_{1}^{2} q_{2} p_{2}+26 p_{1} p_{2} q_{3}+p_{3}^{2}+ \\
& +2 q_{4} p_{1}^{2}+30 p_{3} q_{1} p_{1}^{2}-24 p_{2}^{3}-4 q_{2} p_{4}+30 p_{1} p_{3} q_{2}-6 p_{1} q_{1}^{2} p_{3}-14 q_{3} p_{1}^{2} q_{1}+ \\
& +4 q_{1} q_{2}^{2} p_{1}-38 p_{1} q_{1} q_{2} p_{2}+18 p_{1} p_{4} q_{1}-12 q_{3} p_{2} q_{1}+2 q_{1}^{2} p_{1} q_{3}+36 p_{2} p_{3} q_{1}- \\
& -18 p_{1} p_{2} q_{1}^{3}-12 q_{1} p_{3} q_{2}+36 p_{1} q_{1} p_{2}^{2}+p_{1}^{2}\left(q_{1}-p_{1}\right)^{2}\left(q_{1}-2 p_{1}\right)^{2}-2 q_{2} p_{1}^{4}- \\
& -2 p_{2}^{2} q_{1}^{2}-10 q_{2} q_{3} p_{1}-14 p_{4} p_{1}^{2}+34 q_{2} p_{2}^{2}-16 q_{1}^{2} q_{2} p_{1}^{2}-4 p_{4} q_{1}^{2}+4 q_{1}^{3} q_{2} p_{1}- \\
& -48 p_{1}^{3} p_{2} q_{1}-72 p_{1} p_{2} p_{3}-4 q_{1} q_{4} p_{1}+16 q_{1} q_{2} p_{1}^{3}+50 p_{1}^{2} p_{2} q_{1}^{2}+68 p_{2} q_{2} p_{1}^{2} .
\end{aligned}
$$

Для экономии места мы ввели обозначения $p_{i}=\partial_{x}^{i} p, q_{i}=\partial_{x}^{i} q$.

Другое свойство, обычное для явно решаемых уравнений, - наличие обобщенных симметрий. Обобщенные симметрии высшего уравнения Даламбера можно получить из его интегралов с помощью формулы

$$
u_{\tau}=\left(\frac{n-1}{2} u D_{x}-u_{x}\right) \omega+\left(\frac{n-1}{2} u D_{t}-u_{t}\right) \bar{\omega},
$$

где $\omega$ и $\bar{\omega}-t$ - и $x$-интегралы $(4)$.

\section{3. АНАЛОГИ ОТКРЫТЫХ ЦЕПОЧЕК ТОДЫ}

Выше был поднят вопрос, существуют ли другие цепочки, связанные с уравнением (4) и имеющие достаточно простой вид. Ниже мы приводим два таких примера: цепочка Шабата-Ямилова и иная форма двумерной цепочки Тоды. Нам не известно, упоминалась ли где либо связь между этими цепочками и уравнением (4).

Цепочка

$$
w_{j, t x}=w_{j, t} w_{j, x}\left(\frac{1}{w_{j}-w_{j-1}}-\frac{1}{w_{j+1}-w_{j}}\right)
$$

была введена Шабатом и Ямиловым [6] как один из двумерных аналогов вырождений модели Ландау-Лифшица. Можно проверить, что на решениях (4) величины

$$
w_{j}=\partial_{u} \ln W_{j}(u), \quad j=1, \ldots, n-1,
$$

удовлетворяют уравнениям цепочки (14) вместе с граничными условиями

$$
w_{0}=0, \quad w_{n}=\infty .
$$

Цепочку (14) можно поэтому рассматривать как аналог открытой цепочки Тоды $A_{n}$. У цепочки Шабата-Ямилова есть хорошо известные частные случаи. Например, для $n=3$ имеем вырожденную систему Лунда-Редже (комплексную систему синус-Гордон I)

$$
v_{t x}=\frac{w v_{t} v_{x}}{v w-1}, \quad w_{t x}=\frac{v w_{t} w_{x}}{v w-1},
$$

где $w_{1}=1 / v, w_{2}=w$. Система (16) использовалась в работах [4], [7] как рабочий пример для демонстрации свойств систем типа Лиувилля. 
Заметим, что уравнение (4) допускает редукции, позволяющие построить аналоги цепочки (14), соответствующие алгебрам Ли $C_{n}, B_{n}$ и, возможно, $D_{n}$. Эта задача будет рассмотрена в другом месте. Вместо этого мы дадим один пример такой цепочки, сходный с цепочкой Тоды $C_{2}$. Можно проверить, что уравнение $W_{5}(u)=0$ допускает редукцию $W_{3}(u)=u$. Последнее уравнение можно тогда записать как систему

$$
v_{t x}=\frac{w v_{x} v_{t}}{w v-1}, \quad w_{t x}=\frac{v w_{x} w_{t}}{w v-1}-\frac{(w v-1)^{3} v}{v_{t}^{2} v_{x}^{2}},
$$

где

$$
\frac{1}{v}=\partial_{u} \ln W_{1}(u)=\frac{1}{u}, \quad w=\partial_{u} \ln W_{2}(u)=\frac{u_{t x}}{u_{t x} u-u_{x} u_{t}} .
$$

Еще один пример цепочки, имеющей отношение к уравнению (4), дается формулой

$$
v_{j, t x}=e^{v_{j+1}-v_{j}}-e^{v_{j}-v_{j-1}}, \quad j=1, \ldots, n-1,
$$

с граничными условиями

$$
v_{0}=\infty, \quad v_{n}=-\infty .
$$

Это другое хорошо известное воплощение двумерной цепочки Тоды. Преобразование, связывающее системы (9) и (18), дается формулой

$$
v_{j}=w_{j}-w_{j-1}
$$

С другой стороны, система уравнений (18) связана с уравнением (4) преобразовани$\mathrm{eM}$

$$
v_{j+1}=-\ln \left(\frac{\partial}{u_{j j}} \ln W_{j+1}(u)\right), \quad j=0, \ldots, n-2,
$$

где $u_{j j}=\partial^{2 j} u / \partial t^{j} \partial x^{j}$.

Есть примеры явно разрешимых систем, имеющие отношение к уравнению (4) определенного порядка. Рассмотрим, например, уравнение

$$
W_{4}(u)=0 .
$$

Вводя переменные

$$
m=-2 \ln u, \quad v=-\frac{4}{a} W_{2}(u), \quad w=\frac{a c}{4 u} \frac{\partial \ln W_{3}(u)}{\partial u_{t x}},
$$

можно переписать уравнение (19) в виде системы

$$
m_{t x}=\frac{a}{2} v e^{m}, \quad v_{t x}=\frac{w v_{x} w_{t}}{v w+c}, \quad w_{t x}=\frac{v w_{t} w_{x}}{v w+c}+\frac{a}{4}(v w+c) e^{m} .
$$

Эта система была получена в работе [8] как вырожденный вариант $S$-интегрируемой системы.

Общие решения и интегралы вышеприведенных систем можно легко вывести из формул (5) и (10). Предложенные преобразования, безусловно, не исчерпывают всех возможных связей между уравнениями типа (4) и открытыми двумерными цепочками. 
Благодарности. Автор благодарен В.Е. Адлеру за указание на работу [6] и В. В. Соколову за внимание к этой работе.

\section{Список литературы}

[1] А. Н. Лезнов, М. В. Савельев, Групповые методы интегрирования нелинейных динамических систем, Наука, М., 1985.

[2] А. В. Жибер, В. В. Соколов, УМН, 56:1(337) (2001), 63-106.

[3] А. М. Гурьева, А. В. Жибер, ТМФ, 138:3 (2004), 401-421.

[4] В. В. Соколов, С. Я. Старцев, ТМФ, 155:2 (2008), 344-355.

[5] Шабат А.Б., Ямилов Р. И., Экспоненциальные системъ типа I и матрицы Картана, Препринт, Башкирский филиал АН СССР, Уфа, 1981.

[6] A. B. Shabat, R. I. Yamilov, Phys. Lett. A, 227:1-2 (1997), 15-23.

[7] Д. К. Демской, С. Я. Старцев, Фундамент. и прикл. матем., 10:1 (2004), 29-37.

[8] Д. К. Демской, ТМФ, 141:2 (2004), 208-227. 